\title{
Bekenstein-Hawking Entropy and Strange Metals
}

\author{
Subir Sachdev \\ Department of Physics, Harvard University, Cambridge, Massachusetts 02138, USA \\ Perimeter Institute for Theoretical Physics, Waterloo, Ontario N2L 2Y5, Canada \\ Kavli Institute for Theoretical Physics, University of California, \\ Santa Barbara, California 93106-4030, USA \\ (Received 22 June 2015; published 13 November 2015)
}

\begin{abstract}
We examine models of fermions with infinite-range interactions that realize non-Fermi liquids with a continuously variable $\mathrm{U}(1)$ charge density $\mathcal{Q}$ and a nonzero entropy density $\mathcal{S}$ at vanishing temperature. Real-time correlators of operators carrying U(1) charge $q$ at a low temperature $T$ are characterized by a $\mathcal{Q}$-dependent frequency $\omega_{\mathcal{S}}=(q T / \hbar)(\partial \mathcal{S} / \partial \mathcal{Q})$, which determines a spectral asymmetry. We show that the correlators match precisely with those of the two-dimensional anti-de Sitter $\left(\mathrm{AdS}_{2}\right)$ horizons of extremal charged black holes. On the black hole side, the matching employs $\mathcal{S}$ as the Bekenstein-Hawking entropy density and the laws of black hole thermodynamics that relate $(\partial \mathcal{S} / \partial \mathcal{Q}) /(2 \pi)$ to the electric field strength in $\mathrm{AdS}_{2}$. The fermion model entropy is computed using the microscopic degrees of freedom of a UV complete theory without supersymmetry.
\end{abstract}

DOI: 10.1103/PhysRevX.5.041025

\section{INTRODUCTION}

Holography provides us with powerful tools for investigating models of quantum matter without quasiparticle excitations. The best understood among these are strongly coupled conformal field theories (CFTs) in spatial dimensions $d \geq 2$. Our understanding of such models is built upon the foundation provided by the solvable example of maximally supersymmetric Yang-Mills theory, which is known to be holographically dual to string theory on anti-de Sitter (AdS) space [1].

However, many holographic studies [2-4] have focused on experimentally important examples of strongly coupled quantum matter that are not CFTs. Of particular interest are compressible states without quasiparticles, or "strange metals," in dimensions $d \geq 2$. Broadly defined, these are quantum states without quasiparticles in which a conserved $\mathrm{U}(1)$ charge density $\mathcal{Q}$ can be continuously varied at zero temperature by a conjugate chemical potential, and the $\mathrm{U}(1)$ and translational symmetries are not spontaneously broken. Solvable examples of strange metals with holographic duals would clearly be of great interest.

Here, we consider the strange metal state introduced by Sachdev and Ye (SY) [5] in a model of fermions with infinite-range interactions. The fermion density $\mathcal{Q}$ is conserved and continuously variable, and there is a nonzero entropy density $\mathcal{S}$ at vanishing temperature [6,7]. The

Published by the American Physical Society under the terms of the Creative Commons Attribution 3.0 License. Further distribution of this work must maintain attribution to the author(s) and the published article's title, journal citation, and DOI.
Subject Areas: Condensed Matter Physics,

Particles and Fields fermion Green function is momentum independent and so has no Fermi surface (but there is a remnant of the Luttinger theorem, as discussed in Appendix A). The Green function is divergent at low frequency $(\omega)$ and temperature $(T)$ with a known scaling function [6-8] [the explicit form is in Eq. (3)] determined by the fermion scaling dimension $\Delta$, its $\mathrm{U}(1)$ charge $q=1$, and a spectral asymmetry frequency we shall denote by $\omega_{\mathcal{S}}$. This frequency determines the asymmetry between the particle and hole excitations of the non-Fermi liquid. The values of $\Delta$ and $q$ are fixed and universal (as in traditional critical phenomena), while that of $\omega_{\mathcal{S}}$ varies with the compressible density $\mathcal{Q}$ in an apparently nonuniversal manner. However, the same $\omega_{\mathcal{S}}$, scaled by the value of $q$, appears in the correlators of all operators.

One general way to fix the precise value of $\omega_{\mathcal{S}}$, without a priori knowledge of the full $\omega$ dependence of the correlator, is the following. The product of the retarded $\left(G^{R}\right)$ and advanced $\left(G^{A}\right)$ Green functions obeys

$$
G^{R}(\omega) G^{A}(\omega)=\Phi_{e}\left(\omega-\omega_{\mathcal{S}}\right),
$$

where $\Phi_{e}(\omega)$ is some even function of $\omega$. So the content of Eq. (1) is that $G^{R} G^{A}$ becomes an even function of frequency after the frequency shift $\omega_{\mathcal{S}}$. With this definition, it was found [6-8] that there is a surprising general relationship between $\omega_{\mathcal{S}}$ and the zero-temperature entropy $\mathcal{S}$ density:

$$
\omega_{\mathcal{S}}=\frac{q T}{\hbar} \frac{\partial \mathcal{S}}{\partial \mathcal{Q}} .
$$


Such a relationship was first found in the "multichannel Kondo" problem of a local spin degree of freedom at the boundary of a CFT2 (i.e., a CFT in $1+1$ spacetime dimensions) [6], where $\mathcal{S}$ is the boundary entropy [9-11]. It was later extended $[7,8]$ to the fermion model of SY, in which we define $\mathcal{S}$ and $\mathcal{Q}$ per site, and there are no explicit CFT2 degrees freedom; instead, each fermion site is influenced by a self-consistent environment, and this environment plays a role similar to that of the CFT2 in the Kondo problem. Both $\mathcal{S}$ and $\omega_{\mathcal{S}}$ are nonuniversal functions of the compressible density $\mathcal{Q}$, but they are related as in Eq. (2). It is quite remarkable to have a dynamical frequency determined by a thermodynamic property (which is also defined classically) divided by $\hbar$; other notable instances of connections between observables characterizing low-frequency dissipation and static thermodynamics or fundamental constants are in Refs. [12,13]. For the SY state, this value of $\omega_{\mathcal{S}}$ relies on emergent symmetries at low energies, but also requires careful regularization of the single-site canonical fermions present at high energies. Note that the entropy $\mathcal{S}$ density in Eq. (2) counts all the degrees of freedom in an UV finite fermion model, as does the charge density $\mathcal{Q}$, while $\omega_{\mathcal{S}}$ characterizes the low-frequency dynamics. Indeed, in this context, Parcollet et al. [6] note, "It is tempting to speculate that a deeper interpretation of these facts is still to be found."

As we demonstrate in this paper, the above properties of the SY state match precisely with the quantum theory holographically dual to extremal charged black holes with two-dimensional anti-de Sitter $\left(\mathrm{AdS}_{2}\right)$ horizons [14-17]. As a specific example, we work with the Einstein-Maxwell theory of (planar or spherical) charged black holes embedded in asymptotically $\operatorname{AdS}_{d+2}$ space, with $d \geq 2$ (the Reissner-Nordström-AdS solution); however, the key features apply to a wide class of black hole solutions [18-24]. The correlators of this gravitational theory have the same functional dependence upon $\omega, T, q, \Delta$, and $\omega_{\mathcal{S}}$ as those of the SY state, given in Eq. (3), and this agreement can be understood by the common conformal and gauge invariances of the two theories [25-27]. However, there is a deeper correspondence between the two theories in that Eq. (2) for the value of $\omega_{\mathcal{S}}$ also applies in the gravitational theories. The holographic computation of correlators yields the value of $\omega_{\mathcal{S}}$ [e.g., by using Eq. (1)], while the right-hand side of Eq. (2) is obtained from a classical gravitational computation of the Bekenstein-Hawking (or Wald) entropy. The equality in Eq. (2) follows from the classical general relativity of $\mathrm{AdS}_{2}$ horizons of charged black holes (see Sec. III B), and this potentially provides the long-sought interpretation for the value of $\omega_{\mathcal{S}}$.

For a general black hole solution, the values of $\omega_{\mathcal{S}}$ and $\mathcal{S}$ depend on $\mathcal{Q}$ in a manner different from the SY state, but they all continue to obey Eq. (2). This difference is not surprising, given that the $\mathcal{Q}$ dependence of $\mathcal{S}$ for the SY state uses its canonical site-fermion structure in the UV, a characteristic that is not expected to be captured by a gravity dual. But the validity of Eq. (2) in the SY state, and in a wide class of gravity theories, is evidence that there is a gravity dual that captures all the universal low-energy properties of the SY state.

The common two-point correlator of a fermionic operator with $\mathrm{U}(1)$ charge $q$, and scaling dimension $\Delta$, in both the $\mathrm{SY}$ and $\mathrm{AdS}_{2}$ theories is $[6-8,16,28,29]$

$$
G^{R}(\omega)=G^{A *}(\omega)=\frac{-i C e^{-i \theta}}{(2 \pi T)^{1-2 \Delta}} \frac{\Gamma\left(\Delta-\frac{i \hbar\left(\omega-\omega_{\mathcal{S}}\right)}{2 \pi k_{B} T}\right)}{\Gamma\left(1-\Delta-\frac{i \hbar\left(\omega-\omega_{\mathcal{S}}\right)}{2 \pi k_{B} T}\right)},
$$

where $\Delta=1 / 4$ for the $q=1$ fundamental fermion of the SY state, the amplitude $C$ is a real and positive, and the angle $-\pi \Delta<\theta<\pi \Delta$ is given by

$$
e^{2 \pi q \mathcal{E}}=\frac{\sin (\pi \Delta+\theta)}{\sin (\pi \Delta-\theta)} .
$$

Here, we find it convenient to introduce a dimensionless, $T$-independent, parameter $\mathcal{E}$ related to $\omega_{\mathcal{S}}$ by

$$
\mathcal{E}=\frac{1}{2 \pi q} \frac{\hbar \omega_{\mathcal{S}}}{k_{B} T}
$$

We therefore introduce three parameters, $\omega_{\mathcal{S}}, \mathcal{E}$, and $\theta$, all of which characterize the spectral asymmetry, and they can be determined from each other in Eqs. (4) and (5). The $T \rightarrow 0$ limit of the Fourier transform of Eq. (3) shows that $\mathcal{E}$ also defines a "twist" in the imaginary time fermionic correlator:

$$
G(\tau) \sim \begin{cases}-\tau^{-2 \Delta} & \tau>0 \\ e^{-2 \pi q \mathcal{E}}|\tau|^{-2 \Delta} & \tau<0\end{cases}
$$

It is easy to verify that Eq. (3) obeys Eq. (1). We show a plot of Eq. (3) in Fig. 1, which illustrates the "shift" property of $G^{R}(\omega) G^{A}(\omega)$.

For the SY state, the previous work [6-8] establishes the additional relation in Eq. (2), which now relates the spectral asymmetry parameters $\omega_{\mathcal{S}}, \mathcal{E}$, and $\theta$ to $\partial \mathcal{S} / \partial \mathcal{Q}$. The $T=0$ properties of the SY state reviewed above are summarized in the left-hand panel of Fig. 2. For our subsequent discussion, it is useful to combine Eqs. (2) and (5) in the form

$$
\frac{\partial \mathcal{S}}{\partial \mathcal{Q}}=2 \pi \mathcal{E}
$$

In the holographic computation of Eq. (3), the temperature $T$ is the Hawking temperature of the black hole horizon [30], and the dimensionless spectral asymmetry 


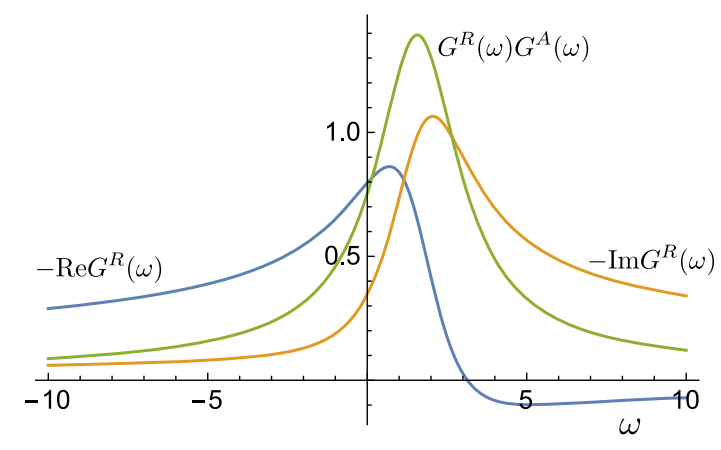

FIG. 1. Plots of the Green functions in Eq. (3) for $\Delta=1 / 4$, $q=1, T=1, A=1, \mathcal{E}=1 / 4$, with $\hbar=k_{B}=1$. Note that while neither $\operatorname{Im} G^{R}(\omega)$ nor $\operatorname{Re} G^{R}(\omega)$ have any definite properties under $\omega \leftrightarrow-\omega$, the product $G^{R}(\omega) G^{A}(\omega)$ becomes an even function of $\omega$ after a shift by $\omega_{\mathcal{S}}=2 \pi q \mathcal{E} T=\pi / 2$.

parameter $\mathcal{E}$ appearing in Eq. (6) [and Eq. (3)] is determined by the strength of the electric field [see Eq. (57)] supporting the near-horizon $\mathrm{AdS}_{2}$ geometry [16,29,31] (see Fig. 2). A key observation in the holographic framework is that $\mathcal{E}$, now related to the electric field, obeys an important identity which follows from the laws of black hole thermodynamics
[32] (see Fig. 2) (we set $\hbar=k_{B}=1$ in the remaining discussion):

$$
\frac{\partial \mathcal{S}_{\mathrm{BH}}}{\partial \mathcal{Q}}=2 \pi \mathcal{E}
$$

where $\mathcal{S}_{\mathrm{BH}}$ is the Bekenstein-Hawking entropy density of the $\mathrm{AdS}_{2}$ horizon. Indeed, Eq. (8) is a general consequence of the classical Maxwell and Einstein equations and the conformal invariance of the $\mathrm{AdS}_{2}$ horizon, as we show in Sec. III B. Moreover, a Legendre transform of the identity in Eq. (8) was established by Sen $[18,19]$ for a wide class of theories of gravity in the Wald formalism [20-24], in which $\mathcal{S}_{\mathrm{BH}}$ is generalized to the Wald entropy.

The main result of this paper is the identical forms of the relationship Eq. (7) for the statistical entropy of the SY state and Eq. (8) for the Bekenstein-Hawking entropy of $\mathrm{AdS}_{2}$ horizons. This result is further evidence that the SY state and a gravity dual with a $\mathrm{AdS}_{2}$ horizon share the same low-energy properties. Assuming the existence of a gravity dual, Eqs. (7) and (8) show that such a correspondence is consistent only if the black hole entropy has the
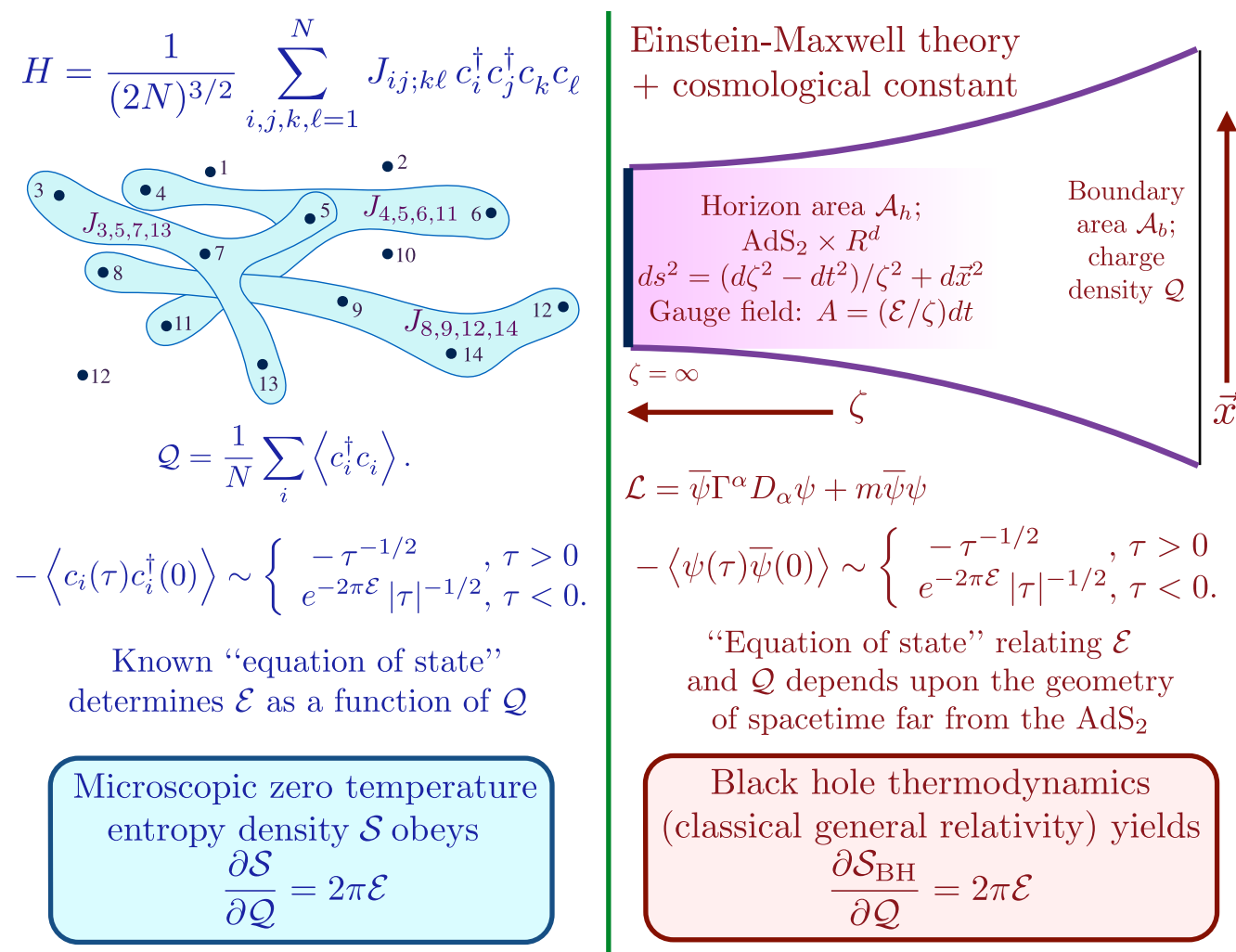

FIG. 2. Summary of the properties of the SY state (Sec. II) and planar charged black holes (Sec. III) at $T=0$. The spatial coordinate $\vec{x}$ has $d$ dimensions. All results also apply to spherical black holes considered in Appendix B. The AdS $\mathrm{S}_{2} \times R^{d}$ metric has unimportant prefactors noted in Eq. (55), which are not displayed in the figure. The fermion mass $m$ has to be adjusted to obtain the displayed power law. The spectral asymmetry parameter $\mathcal{E}$ appears in the fermion correlators and in the $\mathrm{AdS}_{2}$ electric field. As the charge $\mathcal{Q}$ is increased, the horizon moves closer to the boundary, and its area $\mathcal{A}_{h}$ increases. In black hole thermodynamics, the Bekenstein-Hawking entropy density $\mathcal{S}_{\mathrm{BH}}$ is related to the area of the horizon via $\mathcal{S}_{\mathrm{BH}}=\mathcal{A}_{h} /\left(4 G_{N} \mathcal{A}_{b}\right)$, where $G_{N}$ is Newton's constant. 
Bekenstein-Hawking value, and endow the black hole entropy with a statistical interpretation [33].

It is important to keep in mind that (as we mentioned earlier) the models considered here have a different "equation of state" relating $\mathcal{E}$ to $\mathcal{Q}$ : this is specified for the SY state in Eq. (A5), for the planar black hole in Eq. (58), and for the spherical black hole in Eq. (B8).

The holographic link between the SY state and the $\mathrm{AdS}_{2}$ horizons of charged black branes has been conjectured earlier [25-27], based upon the presence of a nonvanishing zero-temperature entropy density and the conformal structure of correlators. The results above sharpen this link by establishing a precise quantitative connection for the Bekenstein-Hawking entropy $[34,35]$ of the black hole with the UV complete computation on the microscopic degrees of freedom of the SY state.

It also worthwhile to note here that in the usual matrix large- $M$ limit of the AdS/CFT correspondence [1], $\mathcal{S}$ and $\mathcal{Q}$ are both of order $M^{2}$ [16]. So $\omega_{\mathcal{S}}$ and $\mathcal{E}$ both remain of order unity in this limit.

We present an infinite-range model and its solution in Sec. II. An important result here is the emergent conformal and gauge invariance in Eq. (26), which strongly constrains the low-energy theory. We then turn to the EinsteinMaxwell theory of charged horizons in Sec. III and show that it also obeys Eqs. (1)-(8). We conclude with a discussion of broader implications in Sec. IV.

\section{INFINITE-RANGE MODEL}

SY considered a model of $\mathrm{SU}(M)$ spins with Gaussian random exchange interactions between any pair of $N$ sites, followed by the double limit $N \rightarrow \infty$ and then $M \rightarrow \infty$. Their Hamiltonian is

$$
H=\frac{1}{(N M)^{1 / 2}} \sum_{i, j=1}^{N} \sum_{\alpha, \beta=1}^{M} J_{i j} c_{i \alpha}^{\dagger} c_{i \beta} c_{j \beta}^{\dagger} c_{j \alpha},
$$

where the $c_{i \alpha}$ are canonical fermions obeying

$$
c_{i \alpha} c_{j \beta}+c_{j \beta} c_{i \alpha}=0, \quad c_{i \alpha} c_{j \beta}^{\dagger}+c_{j \beta}^{\dagger} c_{i \alpha}=\delta_{i j} \delta_{\alpha \beta},
$$

and there is a fermion number constraint,

$$
\frac{1}{M} \sum_{\alpha} c_{i \alpha}^{\dagger} c_{i \alpha}=\mathcal{Q}
$$

on every site $i$, with $0<\mathcal{Q}<1$. The exchange interactions $J_{i j}$ are independent Gaussian random numbers with zero mean and equal variance.

Kitaev [27] recently pointed out that the SY state can also be realized in a simpler model of Majorana fermions in which only a single large- $N$ limit needs to be taken, and which also suppresses spin-glass order [7,36-38]. We present our results here using a complex fermion generalization of Kitaev's proposal, but we emphasize that essentially all results below apply equally to the original model of SY in Eq. (9). We consider the Hamiltonian of spinless fermions $c_{i}$,

$$
H=\frac{1}{(2 N)^{3 / 2}} \sum_{i, j, k, \ell=1}^{N} J_{i j ; k \ell} c_{i}^{\dagger} c_{j}^{\dagger} c_{k} c_{\ell}-\mu \sum_{i} c_{i}^{\dagger} c_{i},
$$

with

$$
c_{i} c_{j}+c_{j} c_{i}=0, \quad c_{i} c_{j}^{\dagger}+c_{j}^{\dagger} c_{i}=\delta_{i j},
$$

and the $J_{i j ; k \ell}$ are complex, independent Gaussian random couplings with zero mean obeying

$$
\begin{aligned}
J_{j i ; k \ell} & =-J_{i j ; k \ell}, & J_{i j ; \ell k} & =-J_{i j ; k \ell}, \\
J_{k \ell ; i j} & =J_{i j ; k \ell}^{*}, & \overline{\left|J_{i j ; k \ell}\right|^{2}} & =J^{2} .
\end{aligned}
$$

Because there is only a fermion interaction term in $H$, and no fermion hopping, Eq. (12) can be viewed as a "matrix model" on Fock space, with a dimension that is exponentially large $N$. The conserved $\mathrm{U}(1)$ density $\mathcal{Q}$ is now related to the average fermion number by

$$
\mathcal{Q}=\frac{1}{N} \sum_{i}\left\langle c_{i}^{\dagger} c_{i}\right\rangle,
$$

which replaces the on-site constraint in Eq. (11). The value of $0<\mathcal{Q}<1$ can be varied by the chemical potential $\mu$. The solution described below applies for any $\mu$, and so realizes a compressible state.

Note that we could equally have defined $\mathcal{Q}$ without the $1 / N$ prefactor in Eq. (15); then we would have to define $\mathcal{S}$ as the total entropy, and both $\mathcal{Q}$ and $\mathcal{S}$ would be proportional to $N$ in the large- $N$ limit. The latter scaling would then be similar to the $M^{2}$ scaling of these quantities in the usual matrix large- $M$ limit of the AdS/CFT correspondence [1]. But we choose to work here with an intensive definition of $\mathcal{Q}$ and $\mathcal{S}$ and keep the $1 / N$ in Eq. (15).

Introducing replicas $c_{i a}$, with $a=1, \ldots, n$, we can average over disorder and obtain the replicated imaginary time $(\tau)$ action:

$$
\begin{aligned}
S= & \sum_{i a} \int_{0}^{1 / T} d \tau c_{i a}^{\dagger}\left(\frac{\partial}{\partial \tau}-\mu\right) c_{i a} \\
& -\frac{J^{2}}{4 N^{3}} \sum_{a b} \int_{0}^{1 / T} d \tau d \tau^{\prime}\left|\sum_{i} c_{i a}^{\dagger}(\tau) c_{i b}\left(\tau^{\prime}\right)\right|^{4}
\end{aligned}
$$

(here, we are neglecting normal-ordering corrections which vanish as $N \rightarrow \infty$ ). Following SY, we decouple the interaction by two successive Hubbard-Stratonovich transformations. First, we introduce the real field $Q_{a b}\left(\tau, \tau^{\prime}\right)$ obeying 


$$
Q_{a b}\left(\tau, \tau^{\prime}\right)=Q_{b a}\left(\tau^{\prime}, \tau\right) .
$$

In terms of this field,

$$
\begin{aligned}
S= & \sum_{i a} \int_{0}^{1 / T} d \tau c_{i a}^{\dagger}\left(\frac{\partial}{\partial \tau}-\mu\right) c_{i a} \\
& +\sum_{a b} \int_{0}^{1 / T} d \tau d \tau^{\prime}\left\{\frac{N}{4 J^{2}}\left[Q_{a b}\left(\tau, \tau^{\prime}\right)\right]^{2}\right. \\
& \left.-\frac{1}{2 N} Q_{a b}\left(\tau, \tau^{\prime}\right)\left|\sum_{i} c_{i a}^{\dagger}(\tau) c_{i b}\left(\tau^{\prime}\right)\right|^{2}\right\} .
\end{aligned}
$$

A second decoupling with the complex field $P_{a b}\left(\tau, \tau^{\prime}\right)$ obeying

$$
P_{a b}\left(\tau, \tau^{\prime}\right)=P_{b a}^{*}\left(\tau^{\prime}, \tau\right)
$$

yields

$$
\begin{aligned}
S= & \sum_{i a} \int_{0}^{1 / T} d \tau c_{i a}^{\dagger}\left(\frac{\partial}{\partial \tau}-\mu\right) c_{i a} \\
& +\sum_{a b} \int_{0}^{1 / T} d \tau d \tau^{\prime}\left\{\frac{N}{4 J^{2}}\left[Q_{a b}\left(\tau, \tau^{\prime}\right)\right]^{2}\right. \\
& +\frac{N}{2} Q_{a b}\left(\tau, \tau^{\prime}\right)\left|P_{a b}\left(\tau, \tau^{\prime}\right)\right|^{2} \\
& \left.-Q_{a b}\left(\tau, \tau^{\prime}\right) P_{b a}\left(\tau^{\prime}, \tau\right) \sum_{i} c_{i a}^{\dagger}(\tau) c_{i b}\left(\tau^{\prime}\right)\right\} .
\end{aligned}
$$

Now we can integrate out the fermions and obtain an action that can be solved in the saddle-point approximation in the limit of large $N$. The saddle-point equations are

$$
\begin{aligned}
P_{a b}\left(\tau, \tau^{\prime}\right) & =\left\langle c_{a}^{\dagger}(\tau) c_{b}\left(\tau^{\prime}\right)\right\rangle, \\
Q_{a b}\left(\tau, \tau^{\prime}\right) & =J^{2}\left|P_{a b}\left(\tau, \tau^{\prime}\right)\right|^{2} .
\end{aligned}
$$

Note that we have dropped the site index on the fermions, because all sites are equivalent and the saddle-point equations are defined as a single-site problem.

We do not expect spin-glass solutions in this model, and so we restrict our attention to replica diagonal solutions in which

$$
P_{a b}\left(\tau, \tau^{\prime}\right)=\delta_{a b} G\left(\tau^{\prime}-\tau\right),
$$

where $G(\tau)$ is the usual fermion Green function. In the operator formalism for the underlying Hamiltonian, this Green function is defined in Euclidean time by

$$
G\left(\tau_{1}, \tau_{2}\right)=-\frac{1}{N} \sum_{i}\left\langle T_{\tau}\left(c_{i}\left(\tau_{1}\right) c_{i}^{\dagger}\left(\tau_{2}\right)\right)\right\rangle,
$$

where $T_{\tau}$ denotes time ordering, and $G\left(\tau_{1}-\tau_{2}\right)=G\left(\tau_{1}, \tau_{2}\right)$. Now, the large- $N$ saddle-point equations become [5]

$$
\begin{aligned}
G\left(i \omega_{n}\right) & =\frac{1}{i \omega_{n}+\mu-\Sigma\left(i \omega_{n}\right)}, \\
\Sigma(\tau) & =-J^{2} G^{2}(\tau) G(-\tau),
\end{aligned}
$$

where $\omega_{n}$ is a Matsubara frequency. Although they are innocuously simple in appearance, these equations contain a great deal of emergent scaling structure.

It was argued in Ref. [5] that physically sensible solutions of Eq. (24) required $\Sigma\left(i \omega_{n}=0\right)=\mu$. Also, in the low-energy scaling limit, $\omega, T \ll J$, the $i \omega_{n}$ is irrelevant [5]. Then, it is useful to write these equations in imaginary (Euclidean) time, separating the two time arguments of the Green functions [and defining $\tilde{\Sigma}\left(i \omega_{n}\right)=\Sigma\left(i \omega_{n}\right)-\mu$ ]:

$$
\begin{aligned}
\int d \tau_{2} G\left(\tau_{1}, \tau_{2}\right) \tilde{\Sigma}\left(\tau_{2}, \tau_{3}\right) & =-\delta\left(\tau_{1}-\tau_{3}\right) \\
\tilde{\Sigma}\left(\tau_{1}, \tau_{2}\right) & =-J^{2}\left[G\left(\tau_{1}, \tau_{2}\right)\right]^{2} G\left(\tau_{2}, \tau_{1}\right)
\end{aligned}
$$

A crucial property of these equations is that they are invariant under the time reparametrization $\tau \rightarrow \sigma$, under which

$$
\begin{aligned}
\tau & =f(\sigma), \\
G\left(\tau_{1}, \tau_{2}\right) & =\left[f^{\prime}\left(\sigma_{1}\right) f^{\prime}\left(\sigma_{2}\right)\right]^{-1 / 4} \frac{g\left(\sigma_{1}\right)}{g\left(\sigma_{2}\right)} G\left(\sigma_{1}, \sigma_{2}\right), \\
\tilde{\Sigma}\left(\tau_{1}, \tau_{2}\right) & =\left[f^{\prime}\left(\sigma_{1}\right) f^{\prime}\left(\sigma_{2}\right)\right]^{-3 / 4} \frac{g\left(\sigma_{1}\right)}{g\left(\sigma_{2}\right)} \tilde{\Sigma}\left(\sigma_{1}, \sigma_{2}\right),
\end{aligned}
$$

where $f(\sigma)$ and $g(\sigma)$ are arbitrary functions, corresponding to emergent conformal and $\mathrm{U}(1)$ gauge invariances. The conformal symmetry of the low-energy Green functions has been noted earlier [8,25-27], and in the form in Eq. (26) by Kitaev [27] [without the $g(\sigma)$ factors]. The gauge transformation $g(\sigma)$ is a real number in Euclidean time, but it becomes a conventional U(1) phase factor in Minkowski time. For the original model of SY [5], the gauge invariance was explicitly present in the underlying Hamiltonian. In contrast, our Hamiltonian here in Eq. (12) is not gauge invariant, and only has a global U(1) symmetry; nevertheless, a U(1) gauge invariance emerges in the low-energy theory.

Note that the $i \omega_{n}$ term in Eq. (24) breaks both the conformal and gauge invariances. Although the $i \omega_{n}$ can mostly be neglected in studying the scaling limit, it is important in selecting the proper low-energy solution of Eq. (25) from the highly degenerate possibilities allowed by Eq. (26).

\section{A. Low-energy Green function}

We now show that the fermion Green function in Eq. (3) follows directly from the conformal and gauge invariances in Eq. (26), when combined with constraints from analyticity and unitarity. This Green function was obtained 
earlier [5-8] by explicit solution of the integral equation in Eq. (25) (and also, as discussed in Sec. III, by the solution $[16,29]$ of a Dirac equation on a thermal $\mathrm{AdS}_{2}$ background with a nonzero electric field). Given our reliance on conformal and gauge invariances, the computations below are straightforwardly generalized to other operators with different values of $q$.

At the Matsubara frequencies, the Green function is defined by

$$
G\left(i \omega_{n}\right)=\int_{0}^{1 / T} d \tau e^{i \omega_{n} \tau} G(\tau)
$$

and this is continued to all complex frequencies $z$ via the spectral representation

$$
G(z)=\int_{-\infty}^{\infty} \frac{d \Omega}{\pi} \frac{\rho(\Omega)}{z-\Omega} .
$$

The spectral density $\rho(\Omega)>0$ for all real $\Omega$ and $T$. The retarded Green function is $G^{R}(\omega)=G(\omega+i \eta)$, with $\eta$ a positive infinitesimal, while the advanced Green function is $G^{A}(\omega)=G(\omega-i \eta)$.

At $T=0$, given the scale invariance implicit in Eq. (26), we expect $G(z)$ to be a power law of $z$. More precisely, Eq. (26) implies

$$
\begin{gathered}
G(z)=C \frac{e^{-i(\pi / 4+\theta)}}{\sqrt{z}}, \quad \operatorname{Im}(z)>0, \\
|z| \ll J, \quad T=0 .
\end{gathered}
$$

Positivity of $\rho(\Omega)$ now implies $C>0$ and $-\pi / 4<\theta<\pi / 4$. An inverse Fourier transform yields

$$
G(\tau)= \begin{cases}-\frac{C \sin (\pi / 4+\theta)}{\sqrt{\pi \tau}} & \tau \gg 1 / J, T=0 \\ \frac{C \cos (\pi / 4+\theta)}{\sqrt{-\pi \tau}} & -\tau \gg 1 / J, T=0\end{cases}
$$

We obtain the nonzero-temperature solution by choosing the conformal map in Eq. (26) as

$$
\tau=\frac{1}{\pi T} \tan (\pi T \sigma),
$$

where $\sigma$ is the periodic imaginary time coordinate with period $1 / T$. Applying this map to Eq. (30), we obtain

$G(\sigma)= \begin{cases}-C g(\sigma) \sin (\pi / 4+\theta)\left(\frac{T}{\sin (\pi T \sigma)}\right)^{1 / 2} & 0<\sigma<\frac{1}{T} \\ C g(\sigma) \cos (\pi / 4+\theta)\left(\frac{T}{\sin (-\pi T \sigma)}\right)^{1 / 2} & 0<-\sigma<\frac{1}{T} .\end{cases}$
The function $g(\sigma)$ is so far undetermined apart from a normalization choice $g(0)=1$. We can now determine $g(\sigma)$ by imposing the Kubo-Martin-Schwinger (KMS) condition

$$
G(\sigma+1 / T)=-G(\sigma),
$$

which implies

$$
g(\sigma)=\tan (\pi / 4+\theta) g(\sigma+1 / T) .
$$

The solution is clearly

$$
g(\sigma)=e^{-2 \pi \mathcal{E} T \sigma},
$$

where the new parameter $\mathcal{E}$ and the angle $\theta$ are related as in Eq. (4) for $\Delta=1 / 4$ and $q=1$. The final expression determining $G(\sigma)$ is

$G(\sigma)=-C \frac{e^{-2 \pi \mathcal{E} T \sigma}}{\sqrt{1+e^{-4 \pi \mathcal{E}}}}\left(\frac{T}{\sin (\pi T \sigma)}\right)^{1 / 2}, \quad 0<\sigma<\frac{1}{T}$,

and this can be extended to all real $\sigma$ using the KMS condition. The result in Eq. (3) now follows from a Fourier transform.

For other fermionic operators with general charge $q$ and scaling dimension $\Delta$, the above arguments show that the $\sigma$ dependence of Eq. (36) will be replaced by

$$
G(\sigma) \sim-e^{-2 \pi q \mathcal{E} T \sigma}\left(\frac{T}{\sin (\pi T \sigma)}\right)^{2 \Delta}, \quad 0<\sigma<\frac{1}{T},
$$

and its Fourier transform will have the frequency shift

$$
\omega_{\mathcal{S}}=2 \pi q T \mathcal{E} .
$$

The $T \rightarrow 0$ limit of Eq. (37) leads to Eq. (6).

The above analysis can be easily repeated for bosonic operators of charge $q$ and scaling dimension $\Delta$. The result in Eq. (3) continues to apply, while Eq. (4) is modified to

$$
e^{2 \pi q \mathcal{E}}=-\frac{\sin (\pi \Delta+\theta)}{\sin (\pi \Delta-\theta)} .
$$

The constraint on the allowed values of $\theta$ is now $\pi \Delta<\theta<\pi(1-\Delta)$.

The constants $C$ and $\theta$ (or $\mathcal{E}$ ) appearing in Eq. (3) can also be determined exactly for the microscopic model in Eq. (12), as reviewed in Appendix A; however, their values depend upon the specific UV completion used here, and do not apply to the holographic model of Sec. III. In particular, the equation of state for $\mathcal{Q}$ as a function of $\mathcal{E}$ is in Eq. (A5). 


\section{B. Entropy}

To complete our results for the SY state, we need to establish the connection in Eq. (2) between $\omega_{\mathcal{S}}$ and the zero-temperature entropy density $\mathcal{S}$. This is connection is the focus of our work, and it also relies on the conformal and gauge invariances in Eq. (26). However, in addition, we need information on the UV complete nature of the fermion model and, in particular, the fact that the short-time behavior of the fermion Green function is determined by canonical fermions obeying the anticommutation relations in Eq. (13).

The computation of the entropy follows Refs. [6,7] and relies on the thermodynamic Maxwell relation

$$
\left(\frac{\partial \mathcal{S}}{\partial \mathcal{Q}}\right)_{T}=-\left(\frac{\partial \mu}{\partial T}\right)_{\mathcal{Q}}
$$

In the $T \rightarrow 0$ limit, Parcollet et al. [6] (Sec. VI A 2) show that the right-hand side of Eq. (40) can be evaluated using the imaginary time Green function, and we review their computation here. Their argument requires not only the scaling behavior of the Green function at times $\tau \gg 1 / J$ given in Eq. (37), but also the short-time behavior that is beyond the conformal regime. First, we observe from Eq. (24) the large frequency behavior

$$
G\left(i \omega_{n}\right)=\frac{1}{i \omega_{n}}-\frac{\mu}{\left(i \omega_{n}\right)^{2}}+\cdots
$$

which implies, from Eq. (28),

$$
\mu=-\int_{-\infty}^{\infty} \frac{d \Omega}{\pi} \Omega \rho(\Omega)
$$

which makes it evident that $\mu$ depends only upon the particle-hole asymmetric part of the spectral density. Next, we can relate the $\Omega$ integrals to the derivative of the imaginary time correlator:

$$
\mu=-\partial_{\tau} G\left(\tau=0^{+}\right)-\partial_{\tau} G\left(\tau=(1 / T)^{-}\right) .
$$

Making the analogy to Eq. (36), we pull out an explicitly particle-hole asymmetric part of $G(\tau)$ by defining [39]

$$
G(\tau) \equiv e^{-2 \pi \mathcal{E} T \tau} g(\tau), \quad 0<\sigma<\frac{1}{T} .
$$

Note that $\mathcal{E}$ was introduced as a parameter in Eq. (36), and then it appears in Eq. (3) via Eq. (38). We proceed in our computation of $\mu$ by inserting Eq. (44) into Eq. (43) to obtain

$$
\begin{aligned}
\mu= & 2 \pi \mathcal{E} T\left[G\left(\tau=0^{+}\right)+G\left(\tau=(1 / T)^{-}\right)\right] \\
& -\partial_{\tau} g\left(\tau=0^{+}\right)-e^{-2 \pi \mathcal{E}} \partial_{\tau} g\left(\tau=(1 / T)^{-}\right) .
\end{aligned}
$$

For the term in the first curly braces, we have

$$
\begin{aligned}
G\left(\tau=0^{+}\right)+G\left[\tau=(1 / T)^{-}\right] & =G\left(\tau=0^{+}\right)-G\left(\tau=0^{-1}\right) \\
& =-1,
\end{aligned}
$$

which follows from the KMS condition and the fermion anticommutation relation in Eq. (13); additionally, this is related to the high-frequency behavior $G(|z| \rightarrow \infty)=1 / z$. Writing the second term in Eq. (45) in terms of a spectral density $\rho_{g}(\Omega)$ for $g(\tau)$, we obtain

$$
\mu=-2 \pi \mathcal{E} T-\int_{-\infty}^{\infty} \frac{d \Omega}{\pi} \frac{\Omega\left[\rho_{g}(\Omega)-e^{-2 \pi \mathcal{E}} \rho_{g}(-\Omega)\right]}{1+e^{-\Omega / T}}
$$

[we note that there is a sign error on the right-hand side of Eq. (65) in Ref. [6], and $-\rho_{g}(-\Omega)$ should be $\rho_{g}(-\Omega)$ ]. At this point, Ref. [6] argues that at low $T$ and fixed $\mathcal{Q}, \rho_{g}$ must be particle-hole symmetric with $\rho_{g}(\Omega)=\rho_{g}(-\Omega)$, and that the $T$-dependent part of the integral above scales as $T^{3 / 2}$. We therefore have

$$
\left(\frac{\partial \mu}{\partial T}\right)_{\mathcal{Q}}=-2 \pi \mathcal{E}, \quad T \rightarrow 0
$$

and then the Maxwell relation in Eq. (40) leads to Eq. (8).

Using the relationship between $\mathcal{Q}$ and $\mathcal{E}$ specified in Appendix A in Eq. (A5), and the limiting value $\mathcal{S}=0$ in the empty state $\mathcal{Q}=0$, we can integrate Eq. (8) to obtain the full zero-temperature entropy [7].

\section{CHARGED BLACK HOLES}

This section (apart from Sec. III B) mainly recalls the results of Faulkner et al. [16,29] on planar, charged black holes in $\operatorname{AdS}_{d+2}$ and makes the correspondence with the properties of the SY state. We also largely follow their notation, apart from the change $d \rightarrow d+1$ required by our definition of $d$ as the spatial dimension (instead of the spacetime dimension). The case of spherical black holes in global AdS is more complicated and is considered in Appendix B; it has a more complex equation of state, but also obeys all results claimed in Sec. I. The discussion in the latter part of Sec. III B shows how the needed features of Faulkner et al. can be obtained in a more general class of black hole solutions.

We consider the Einstein-Maxwell theory of a metric $g$ and a $\mathrm{U}(1)$ gauge flux $F=d A$ with action

$$
S=\frac{1}{2 \kappa^{2}} \int d^{d+2} x \sqrt{-g}\left[\mathcal{R}+\frac{d(d+1)}{R^{2}}-\frac{R^{2}}{g_{F}^{2}} F^{2}\right],
$$

where $\kappa^{2}=8 \pi G_{N}, \mathcal{R}$ is the Ricci scalar, $R$ is the radius of $\mathrm{AdS}_{d+2}$, and $g_{F}$ is a dimensionless gauge coupling constant. The equations of motion of this action have the solution $[14,15]$ 


$$
d s^{2}=\frac{r^{2}}{R^{2}}\left(-f d t^{2}+d \vec{x}^{2}\right)+\frac{R^{2}}{r^{2}} \frac{d r^{2}}{f}
$$

with

$$
\begin{aligned}
& f=1+\frac{\Theta^{2}}{r^{2 d}}-\left(r_{0}^{d+1}+\frac{\Theta^{2}}{r_{0}^{d-1}}\right) \frac{1}{r^{d+1}}, \\
& A=\mu\left(1-\frac{r_{0}^{d-1}}{r^{d-1}}\right) d t .
\end{aligned}
$$

This solution is expressed in terms of three parameters, $\Theta$, $r_{0}$, and $\mu$. These parameters are determined by the charge density $\mathcal{Q}$ and temperature $T$ of the boundary theory via the relations

$$
\begin{aligned}
\mu & =\frac{g_{F} \Theta}{c_{d} R^{2} r_{0}^{d-1}}, \quad \mathcal{Q}=\frac{2(d-1)}{c_{d}} \frac{\Theta}{\kappa^{2} R^{d} g_{F}}, \\
T & =\frac{(d+1) r_{0}}{4 \pi R^{2}}\left(1-\frac{(d-1) \Theta^{2}}{(d+1) r_{0}^{2 d}}\right), \\
c_{d} & =\sqrt{\frac{2(d-1)}{d}}
\end{aligned}
$$

The Bekenstein-Hawking entropy density [34,35] of this solution is

$$
\mathcal{S}_{\mathrm{BH}}=\frac{2 \pi}{\kappa^{2}}\left(\frac{r_{0}}{R}\right)^{d}
$$

We now turn to the holographic implications of this solution at low energy $[16,29,40]$, which is controlled by the near-horizon geometry. At $T=0$, the horizon is at $r=\left[\Theta^{2}(d-1) /(d+1)\right]^{1 /(2 d)}$, and so we introduce the coordinate $\zeta$ by

$$
r-\left[\Theta^{2}(d-1) /(d+1)\right]^{1 /(2 d)}=\frac{1}{\zeta} .
$$

We approach the horizon as $\zeta \rightarrow \infty$ (see Fig. 2). In terms of $\zeta$, the near-horizon geometry at $T=0$ is

$$
d s^{2}=R_{2}^{2} \frac{\left(-d t^{2}+d \zeta^{2}\right)}{\zeta^{2}}+\frac{\left[\Theta^{2}(d-1) /(d+1)\right]^{1 / d}}{R^{2}} d \vec{x}^{2} .
$$

The geometry has factorized to $\mathrm{AdS}_{2} \times \mathbb{R}^{d}$, where the $\mathrm{AdS}_{2}$ radius is given by

$$
R_{2}=\frac{R}{\sqrt{d(d+1)}} .
$$

In the same low-energy limit, the gauge field is (see Fig. 2)

$$
A=\frac{\mathcal{E}}{\zeta} d t
$$

which determines the strength of the $\mathrm{AdS}_{2}$ electric field in terms of the dimensionless parameter $\mathcal{E}$. Notice that the value of $\mathcal{E}$ in Eq. (57) is invariant under any rescaling of the coordinates, which preserves the $\left(-d t^{2}+d \zeta^{2}\right) / \zeta^{2}$ structure of the $\mathrm{AdS}_{2}$ metric. From the present near-horizon computation, we find the value

$$
\mathcal{E}=\frac{g_{F} \operatorname{sgn}(\mathcal{Q})}{\sqrt{2 d(d+1)}}
$$

Equation (58) is the equation of state connecting $\mathcal{Q}$ to $\mathcal{E}$, and the analogous expression for the fermion model is in Eq. (A5), and for the spherical black hole is in Eq. (B8); the nonanalytic $\mathcal{Q}$ dependence in Eq. (58) becomes analytic for the spherical black hole in Appendix B. We recall that $g_{F}$ is a dimensionless coupling, and so $\mathcal{E}$ is also dimensionless, and depends only upon $g_{F}$ and $d$; in particular, $\mathcal{E}$ is independent of $\kappa^{2}$, and so remains of order unity in the matrix large- $M$ limit of holography [1], as noted in Sec. I.

We also take the $T=0$ limit of Eq. (53) from Eq. (52), and find

$$
\mathcal{S}_{\mathrm{BH}}=\frac{2 \pi g_{F}|\mathcal{Q}|}{\sqrt{2 d(d+1)}}, \quad T \rightarrow 0 .
$$

Comparing Eqs. (58) and (59), we find that Eq. (8) is indeed obeyed. Note that for the present case of a planar black hole, we can combine Eqs. (58) and (59) into the simple relationship [16]

$$
\mathcal{S}_{\mathrm{BH}}=2 \pi \mathcal{Q E} .
$$

Equation (60) does not hold for a spherical black hole, but the more fundamental relation for $\partial \mathcal{S}_{\mathrm{BH}} / \partial \mathcal{Q}$ in Eq. (8) does hold, and is verified in Appendix B, which also derives the different equation of state relating $\mathcal{E}$ and $\mathcal{Q}$ for a spherical black hole.

\section{A. Fermion correlations}

To confirm the link to the fermion model, we need to show that the $\mathcal{E}$ obtained in Eq. (57) is the same as the $\mathcal{E}$ [or the related $\omega_{\mathcal{S}}$ via Eq. (5)] appearing as the spectral asymmetry parameter in the response functions in Eqs. (3) and (6) (see Fig. 2). For this, we need the Green function of matter fields moving on a thermal $\mathrm{AdS}_{2}$ metric. The finite-temperature generalization of the $\mathrm{AdS}_{2}$ factor in Eq. (55) is [16,29]

$$
d s^{2}=\frac{R_{2}^{2}}{\zeta^{2}}\left[-\left(1-\zeta^{2} / \zeta_{0}^{2}\right) d t^{2}+\frac{d \zeta^{2}}{\left(1-\zeta^{2} / \zeta_{0}^{2}\right)}\right]
$$

and that of the gauge field is 


$$
A=\mathcal{E}\left(\frac{1}{\zeta}-\frac{1}{\zeta_{0}}\right) d t
$$

where

$$
T=\frac{1}{2 \pi \zeta_{0}}
$$

The action of a fermionic spinor $\psi$ of charge $q$ moving in the backgrounds of Eqs. (61) and (62) is

$$
S=i \int d^{2} x \sqrt{-g}\left(\bar{\psi} \Gamma^{\alpha} D_{\alpha} \psi-m \bar{\psi} \psi\right)
$$

where $m$ is a bulk fermion mass, $\Gamma^{\alpha}$ are the Dirac Gamma matrices, and $D_{\alpha}$ is a covariant derivative with charge $q$. The correlator of $\psi$ in this thermal $\mathrm{AdS}_{2}$ [28] plus electric field background has been computed in some detail by Faulkner et al. [16,29], and their result was already displayed in Eq. (3) in our notation. This computation shows that $\mathcal{E}=\omega_{\mathcal{S}} /(2 \pi q T)$ [Eq. (5)] is indeed the same parameter appearing in Eqs. (57) and (62). In this $\mathrm{AdS}_{2}$ computation, the scaling dimension $\Delta$ is related to the bulk spinor mass by

$$
\Delta=\frac{1}{2}-\sqrt{m^{2} R_{2}^{2}-q^{2} \mathcal{E}^{2}}
$$

\section{B. Black hole thermodynamics}

We close this section by noting a significant property of the above solution of classical general relativity at all $T$ and $\mathcal{Q}$. From the laws of black hole thermodynamics [32], we deduce that the horizon area and the chemical potential must obey a thermodynamic Maxwell relation,

$$
\left(\frac{\partial \mathcal{S}_{\mathrm{BH}}}{\partial \mathcal{Q}}\right)_{T}=-\left(\frac{\partial \mu}{\partial T}\right)_{\mathcal{Q}}
$$

which is the analog of that in the fermion model computation in Eq. (40). And indeed we do find from Eqs. (52) and (53) that Eq. (66) is obeyed with

$$
\left(\frac{\partial \mu}{\partial T}\right)_{\mathcal{Q}}=-\frac{4 \pi(d-1) g_{F} \Theta r_{0}^{d}}{c_{d}(d+1) r_{0}^{2 d}+c_{d}(d-1)(2 d-1) \Theta^{2}}
$$

In determining the value of $(\partial \mu / \partial T)_{\mathcal{Q}}$ as $T \rightarrow 0$, rather than explicitly evaluating Eq. (67), it is instructive to use a more general argument that does not use the explicit form of the solution in Eqs. (51) and (52). From the original action in Eq. (49) and the metric in Eq. (50), Gauss's law for the scalar potential in the bulk is

$$
\frac{2 R^{2}}{\kappa^{2} g_{F}^{2}} \frac{d}{d r}\left(\frac{r^{d}}{R^{d}} \frac{d A_{t}}{d r}\right)=0
$$

and the constant of integration is the boundary charge density,

$$
\frac{2 R^{2}}{\kappa^{2} g_{F}^{2}}\left(\frac{r^{d}}{R^{d}} \frac{d A_{t}}{d r}\right)=\mathcal{Q} .
$$

We can write the solution of Eq. (69) as

$$
A_{t}(r)=\mu(T)-\left(\frac{R^{d-2} \kappa^{2} g_{F}^{2}}{2(d-1)}\right) \frac{\mathcal{Q}}{r^{d-1}},
$$

where the $r$-dependent term in Eq. (70) is independent of $T$ at fixed $\mathcal{Q}$, and the chemical potential $\mu$ equals $A_{t}(r \rightarrow \infty)$ when we choose $A_{t}=0$ on the horizon. Now, we transform to the near-horizon $\mathrm{AdS}_{2}$ geometry by making a $T$-independent change of variables from $r$ to $\zeta$ as in Eq. (54), $r=r_{*}+1 / \zeta$, where $r=r_{*}$ is the position of the horizon at $T=0$, but we will not need the actual value of $r_{*}$. Then Eq. (70) implies that, as $\zeta \rightarrow \infty$, the near-horizon scalar potential must be of the form in Eq. (62), where now we define $\zeta=\zeta_{0}$ as the position of the horizon at nonzero $T$, where $\mathcal{E}$ is a parameter independent of $T$, and

$$
\left(\frac{\partial \mu}{\partial T}\right)_{\mathcal{Q}}=\mathcal{E} \frac{\partial}{\partial T}\left(-\frac{1}{\zeta_{0}}\right)_{\mathcal{Q}} .
$$

The $T$ dependence of $\zeta_{0}$ in Eq. (63) follows from the conformal mapping between the $T=0 \mathrm{AdS}_{2}$ metric in Eq. (55) and the $T>0$ metric in Eq. (62) [29,41]. So we find by this general argument that

$$
\left(\frac{\partial \mu}{\partial T}\right)_{\mathcal{Q}}=-2 \pi \mathcal{E}, \quad T \rightarrow 0
$$

which is the same as the fermion model result in Eq. (48). It can be verified that Eq. (72) holds also in the spherical geometry of Appendix B. Combining Eq. (72) with Eq. (66), we obtain Eq. (8), which is a special case of results obtained from the Wald formalism [18-24].

We note that the above derivation of Eq. (72) relies only on Gauss's law and the conformal invariance of the $\mathrm{AdS}_{2}$ near-horizon geometry: this implies that such results hold for a wide class of black hole solutions [18-24].

\section{DISCUSSION}

In our discussion of the SY state of the infinite-range fermion model in Eq. (12), we note that the fermion Green function is almost completely determined by the emergent conformal and gauge invariances in Eq. (26). These conformal and gauge invariances also fairly uniquely determine the holographic theory of matter moving in 
curved space in the presence of an electric field. So, with the benefit of hindsight, we can understand the equivalence of the fermion Green functions obtained in Secs. II and III.

However, we go beyond the identification of the Green functions, and also show that the zero-temperature entropy of the SY state can be mapped onto that of the $\mathrm{AdS}_{2}$ theory (see Fig. 2). Specifically, we choose an appropriate combination of observables in Eqs. (1) and (2) to allow us to generally define a common frequency $\omega_{\mathcal{S}}$, and we show that this frequency is related to precisely the same derivative of the entropy both in the SY state and in charged black holes (where the entropy is the Bekenstein-Hawking entropy). In both cases, establishing this relationship requires an analysis of the details of the model. For the SY state, the entropy computation requires careful treatment of the manner in which the emergent gauge and conformal invariances, present at low energies, are broken by the on-site canonical fermions at high energies, and this consistently removes the cutoff-dependent terms in the chemical potential [39], as discussed in Sec. II B. For the charged black hole, Gauss's law maps the $T$ dependence of the chemical potential to the near-horizon behavior, as discussed in Sec. III B.

This common relationship between $\omega_{\mathcal{S}}$ and the entropy indicates an equivalence between the low-energy degrees of freedom of the two theories in Secs. II and III, and is evidence for the existence of a gravity dual of the SY state with an $\mathrm{AdS}_{2}$ horizon. The present results also imply the $c_{i}$ fermion, with $q=1$, of the theory in Eq. (12) is holographically dual to the $\psi$ fermion, with $q=1[16,40]$ of Eq. (64). As the microscopic $c_{i}$ fermion carries all of the $\mathcal{Q}$ charge of the theory in Eq. (12), we expect that $\psi$ also carries a non-negligible fraction of the charge (in the large$N$ limit) behind the $\mathrm{AdS}_{2}$ horizon. Both models likely also have higher dimension operators, but these have not been analyzed so far (see, however, Ref. [27]).

Note that the above discussion refers to the near-horizon $\mathrm{AdS}_{2}$ geometry. The larger Reissner-Nordström-AdS solution is to be regarded here as a convenient (and nonuniversal) embedding space which provides an UV regulation of the gravitational theory. With such an embedding, we are able to compute well-defined values for $\mathcal{S}$ and $\mathcal{Q}$. Presumably, other gravitational UV embeddings will have different equations of state between $\mathcal{E}$ and $\mathcal{Q}$, but they will nevertheless obey the fundamental relation in Eq. (8) provided they contain an $\mathrm{AdS}_{2}$ horizon. We explicitly test the independence on the UV embedding in Appendix B by comparing the cases of planar and spherical black holes.

The above identification between the $c_{i}$ and $\psi$ fermions differs from that made previously by the author in Refs. $[25,26]$. There, $\psi$ was argued to be dual to a higher dimension composite fermion operator of the original model of SY [5]. This previous identification was based upon the requirement that local bulk operators must be dual to gauge-invariant operators on the boundary, and the original model [5] had a microscopic gauge invariance which did not allow the choice of $c_{i}$ as dual to $\psi$. However, in the present model in Eq. (12), there is no microscopic gauge invariance, and so we are free to use $c_{i}$ as the dual of the bulk $\psi$ field. It turns out that the low-energy boundary theory for $c_{i}$ does have a gauge invariance [as in Eq. (26)], but this is an emergent gauge invariance that is broken by UV terms needed to regularize the theory. The present situation is analogous to the theory of the Ising-nematic quantum critical point in metals, where the regularized model for the electrons is not gauge invariant, but the lowenergy theory defined on two Fermi surface patches does have an emergent gauge structure $[42,43]$. And the present situation is different from that in the "slave particle" theories of condensed matter, where the gauge structure emerges from fractionalizing particles into partons, which influenced the reasoning of Refs. [25,26]. Instead, the same particle can be gauge invariant in the underlying theory and acquire an emergent gauge charge in the low-energy theory. There is some similarity between this interpretation and ideas in Ref. [44].

Finally, we note recent work $[27,45,46]$ on "a bound on chaos" that also related characteristic times of the real-time dynamics of strongly coupled quantum systems to thermodynamics $\hbar$ and black hole horizons.

\section{ACKNOWLEDGMENTS}

I thank T. Banks, A. Dabholkar, Wenbo Fu, S. Hartnoll, C. Herzog, A. Kitaev, Hong Liu, J. McGreevy, R. Myers, A. Sen, A. Strominger, and W. Witczak-Krempa for valuable discussions, and especially $\mathrm{A}$. Georges and $\mathrm{O}$. Parcollet for inspiring discussions on these topics over many years. This research was supported by the NSF under Grant No. DMR-1360789, and also partially by the Templeton Foundation. The research at KITP Santa Barbara was supported by the Simons Foundation and NSF Grant No. PHY11-25915. Research at Perimeter Institute is supported by the Government of Canada through Industry Canada and by the Province of Ontario through the Ministry of Research and Innovation.

\section{APPENDIX A: NONUNIVERSAL CONSTANTS OF THE FERMION MODEL}

We compute the constants $C$ and $\theta$ (or $\mathcal{E}$ ) appearing in Eq. (3) for the microscopic model in Eq. (12). The results of this appendix do not apply to the holographic model of Sec. III.

We can compute the self-energy from Eq. (30) and the second equation in Eq. (24):

$\Sigma(\tau)= \begin{cases}-\frac{C^{3} J^{2} \cos (2 \theta) \sin (\pi / 4+\theta)}{2(\pi \tau)^{3 / 2}} & \tau \gg J, T=0 \\ \frac{C^{3} J^{2} \cos (2 \theta) \cos (\pi / 4+\theta)}{2(-\pi \tau)^{3 / 2}} & -\tau \gg J, T=0 .\end{cases}$

A Fourier transform now leads to 


$$
\begin{gathered}
\Sigma(z)=-\frac{J^{2} C^{3} \cos (2 \theta)}{\pi} e^{i(\pi / 4+\theta)} \sqrt{z}, \\
\operatorname{Im}(z)>0, \quad|z| \ll J, \quad T=0 .
\end{gathered}
$$

We now see that Eqs. (29) and (A2) are consistent with the first equation in Eq. (24), provided we choose the value of $C$ to be

$$
C=\left(\frac{\pi}{J^{2} \cos (2 \theta)}\right)^{1 / 4}
$$

Finally, the value of $\theta$ can be related to the density $\mathcal{Q}$ by a computation which parallels the Luttinger-Ward analysis [47] for a Fermi liquid. The present model has no spatial structure, and so no possibility of a Fermi surface. However, if we apply the steps of the Luttinger-Ward proof of the volume enclosed by the Fermi surface, we find an expression relating density $\mathcal{Q}$ to the spectral asymmetry angle $\theta$. In other words, $\theta$ plays a role similar to the Fermi wave vector in a Fermi liquid. And the relationship between $\mathcal{Q}$ and $\theta$ is [7]

$$
\mathcal{Q}=\frac{1}{2}-\frac{\theta}{\pi}-\frac{\sin (2 \theta)}{4} .
$$

Note that the constraint $-\pi / 4<\theta<\pi / 4$ implies that $0<\mathcal{Q}<1$, as expected. In terms of $\mathcal{E}$, this relationship is

$$
\mathcal{Q}=\frac{1}{4}[3-\tanh (2 \pi \mathcal{E})]-\frac{1}{\pi} \tan ^{-1}\left(e^{2 \pi \mathcal{E}}\right) .
$$

The right-hand side is a monotonically decreasing function of $\mathcal{E}$ that ranges between 1 and 0 , as $\mathcal{E}$ increases from $-\infty$ to $\infty$.

\section{APPENDIX B: SPHERICAL BLACK HOLES}

We consider the case of spherical black holes in global AdS, following the analysis of Ref. [15]. For simplicity, we limit ourselves to the $T=0$ case.

Now, we choose a solution of the Einstein-Maxwell equations of motion of Eq. (49) with metric

$$
d s^{2}=-V(r) d t^{2}+r^{2} d \Omega_{d}^{2}+\frac{d r^{2}}{V(r)},
$$

where $d \Omega_{d}^{2}$ is the metric of the $d$ sphere, and

$$
V(r)=1+\frac{r^{2}}{R^{2}}+\frac{\Theta^{2}}{r^{2 d-2}}-\frac{M}{r^{d-1}}
$$

has a zero at $r=r_{0}$, so that

$$
M=r_{0}^{d-1}\left(1+\frac{r_{0}^{2}}{R^{2}}+\frac{\Theta^{2}}{r_{0}^{2 d-2}}\right) .
$$

The zero-temperature case has [15]

$$
\Theta^{2}=\frac{r_{0}^{2 d-2}\left[(d-1) R^{2}+(d+1) r_{0}^{2}\right]}{(d-1) R^{2}} .
$$

In the near-horizon region, we introduce, as in Sec. III, the coordinate $\zeta$ via

$$
r-r_{0}=\frac{R_{2}^{2}}{\zeta},
$$

where Eq. (56) is now replaced by

$$
R_{2}=\frac{R}{\sqrt{d(d+1)+(d-1)^{2} R^{2} / r_{0}^{2}}},
$$

and the near-horizon metric becomes $\operatorname{AdS}_{2} \times \mathrm{S}_{d}$, with

$$
d s^{2}=R_{2}^{2}\left[\frac{-d t^{2}+d \zeta^{2}}{\zeta^{2}}\right]+r_{0}^{2} d \Omega_{d}^{2}
$$

Turning to the gauge field sector, the charge density $\mathcal{Q}$ and $\mathrm{AdS}_{2}$ electric field parameter $\mathcal{E}$ in Eq. (57) are

$$
\begin{aligned}
\mathcal{Q} & =\frac{r_{0}^{d-1} \sqrt{2 d\left[(d-1) R^{2}+(d+1) r_{0}^{2}\right]}}{\kappa^{2} g_{F}}, \\
\mathcal{E} & =\frac{g_{F} r_{0} \sqrt{2 d\left[(d-1) R^{2}+(d+1) r_{0}^{2}\right]}}{2\left[(d-1)^{2} R^{2}+d(d+1) r_{0}^{2}\right]} .
\end{aligned}
$$

The equation of state obeyed by $\mathcal{E}$ and $\mathcal{Q}$ is obtained by eliminating $r_{0}$ between the equations in Eq. (B8). This leads to a very lengthy expression which we shall not write out explicitly.

Using the Bekenstein-Hawking entropy density,

$$
\mathcal{S}_{\mathrm{BH}}=\frac{2 \pi}{\kappa^{2}} r_{0}^{d},
$$

and

$$
\frac{\partial \mathcal{S}_{\mathrm{BH}}}{\partial \mathcal{Q}}=\frac{\partial \mathcal{S}_{\mathrm{BH}} / \partial r_{0}}{\partial \mathcal{Q} / \partial r_{0}},
$$

and evaluating the derivatives via Eq. (B8), we can now verify that Eq. (8) is indeed obeyed. Note that $\mathcal{S}_{\mathrm{BH}} \neq 2 \pi \mathcal{Q E}$ here, unlike Eq. (60) for the planar case.

[1] J. M. Maldacena, The Large-N Limit of Superconformal Field Theories and Supergravity, Int. J. Theor. Phys. 38, 1113 (1999).

[2] S. A. Hartnoll, Horizons, Holography and Condensed Matter, arXiv:1106.4324. 
[3] S. Sachdev, What Can Gauge-Gravity Duality Teach Us about Condensed Matter Physics?, Annu. Rev. Condens. Matter Phys. 3, 9 (2012).

[4] N. Iqbal, H. Liu, and M. Mezei, Lectures on Holographic Non-Fermi Liquids and Quantum Phase Transitions, in Proceedings of the 2010 Theoretical Advanced Study Institute in Elementary Particle Physics Boulder, Colorado, 2010, edited by M. Dine, T. Banks, and S. Sachdev (World Scientific, Singapore, 2012), pp. 707-815.

[5] S. Sachdev and J. Ye, Gapless Spin-Fluid Ground State in a Random Quantum Heisenberg Magnet, Phys. Rev. Lett. 70, 3339 (1993).

[6] O. Parcollet, A. Georges, G. Kotliar, and A. Sengupta, Overscreened Multichannel SU(N) Kondo Model: Large-N Solution and Conformal Field Theory, Phys. Rev. B 58, 3794 (1998).

[7] A. Georges, O. Parcollet, and S. Sachdev, Quantum Fluctuations of a Nearly Critical Heisenberg Spin Glass, Phys. Rev. B 63, 134406 (2001).

[8] O. Parcollet and A. Georges, Non-Fermi-Liquid Regime of a Doped Mott Insulator, Phys. Rev. B 59, 5341 (1999).

[9] N. Andrei and C. Destri, Solution of the Multichannel Kondo Problem, Phys. Rev. Lett. 52, 364 (1984).

[10] A. M. Tsvelick, The Thermodynamics of Multichannel Kondo Problem, J. Phys. C 18, 159 (1985).

[11] I. Affleck and A. W. W. Ludwig, Universal Noninteger "Ground-State Degeneracy" in Critical Quantum Systems, Phys. Rev. Lett. 67, 161 (1991).

[12] K. Damle and S. Sachdev, Nonzero-Temperature Transport near Quantum Critical Points, Phys. Rev. B 56, 8714 (1997).

[13] P. K. Kovtun, D. T. Son, and A. O. Starinets, Viscosity in Strongly Interacting Quantum Field Theories from Black Hole Physics, Phys. Rev. Lett. 94, 111601 (2005).

[14] L. Romans, Supersymmetric, Cold and Lukewarm Black Holes in Cosmological Einstein-Maxwell Theory, Nucl. Phys. B383, 395 (1992).

[15] A. Chamblin, R. Emparan, C. V. Johnson, and R. C. Myers, Charged AdS Black Holes and Catastrophic Holography, Phys. Rev. D 60, 064018 (1999).

[16] T. Faulkner, H. Liu, J. McGreevy, and D. Vegh, Emergent Quantum Criticality, Fermi Surfaces, and AdS(2), Phys. Rev. D 83, 125002 (2011).

[17] M. Cubrovic, J. Zaanen, and K. Schalm, String Theory, Quantum Phase Transitions and the Emergent FermiLiquid, Science 325, 439 (2009).

[18] A. Sen, Black Hole Entropy Function and the Attractor Mechanism in Higher Derivative Gravity, J. High Energy Phys. 09 (2005) 038.

[19] A. Sen, Entropy Function and AdS(2)/CFT(1) Correspondence, J. High Energy Phys. 11 (2008) 075.

[20] R. M. Wald, Black Hole Entropy Is the Noether Charge, Phys. Rev. D 48, R3427 (1993).

[21] T. Jacobson, G. Kang, and R. C. Myers, On Black Hole Entropy, Phys. Rev. D 49, 6587 (1994).

[22] V. Iyer and R. M. Wald, Some Properties of Noether Charge and a Proposal for Dynamical Black Hole Entropy, Phys. Rev. D 50, 846 (1994).

[23] T. Jacobson, G. Kang, and R. C. Myers, Black Hole Entropy in Higher Curvature Gravity, arXiv:gr-qc/9502009.
[24] M. Johnstone, M. M. Sheikh-Jabbari, J. Simon, and H. Yavartanoo, Extremal Black Holes and the First Law of Thermodynamics, Phys. Rev. D 88, 101503 (2013).

[25] S. Sachdev, Holographic Metals and the Fractionalized Fermi Liquid, Phys. Rev. Lett. 105, 151602 (2010).

[26] S. Sachdev, Strange Metals and the AdS/CFT Correspondence, J. Stat. Mech. (2010) P11022.

[27] A. Y. Kitaev (to be published).

[28] N. Iqbal and H. Liu, Real-Time Response in AdS/CFT with Application to Spinors, Fortschr. Phys. 57, 367 (2009).

[29] T. Faulkner, N. Iqbal, H. Liu, J. McGreevy, and D. Vegh, Holographic Non-Fermi Liquid Fixed Points, Phil. Trans. R. Soc. A 369, 1640 (2011).

[30] G. W. Gibbons and S. W. Hawking, Action Integrals and Partition Functions in Quantum Gravity, Phys. Rev. D 15, 2752 (1977).

[31] T. Hartman and A. Strominger, Central Charge for AdS(2) Quantum Gravity, J. High Energy Phys. 04 (2009) 026.

[32] J. M. Bardeen, B. Carter, and S. W. Hawking, The Four Laws of Black Hole Mechanics, Commun. Math. Phys. 31, 161 (1973).

[33] A. Strominger and C. Vafa, Microscopic Origin of the Bekenstein-Hawking Entropy, Phys. Lett. B 379, 99 (1996).

[34] J. D. Bekenstein, Black Holes and Entropy, Phys. Rev. D 7, 2333 (1973).

[35] S. W. Hawking, Particle Creation by Black Holes, Commun. Math. Phys. 43, 199 (1975).

[36] A. Georges, O. Parcollet, and S. Sachdev, Mean Field Theory of a Quantum Heisenberg Spin Glass, Phys. Rev. Lett. 85, 840 (2000).

[37] L. Arrachea and M. J. Rozenberg, Infinite-Range Quantum Random Heisenberg Magnet, Phys. Rev. B 65, 224430 (2002).

[38] A. Camjayi and M. J. Rozenberg, Quantum and Thermal Fluctuations in the SU(N) Heisenberg Spin-Glass Model near the Quantum Critical Point, Phys. Rev. Lett. 90, 217202 (2003).

[39] It might appear that we can absorb the chemical potential $\mu$ in the Hamiltonian by a temporal gauge transformation, and that such a transformation implies that the frequencies of the Green functions shift via $\omega \rightarrow \omega-q \mu$; such a shift combined with Eq. (44) implies $\mu=-2 \pi \mathcal{E} T$ and so yields $\partial \mu / \partial T$. However, such a gauge transformation does not yield the $\mu$ dependence of correlators in compressible quantum systems: it fails in superfluids and in Fermi liquids, and the gauge invariance is, in a sense, "broken" by the compressible ground state. In the present system, the correct value of $\mu$ is specified by the low- $T$ expansion $\mu=J f(\mathcal{Q})-2 \pi \mathcal{E} T+\cdots$, where $f(\mathcal{Q})$ is a UV-dependent function of $\mathcal{Q}$ which has to be determined numerically, and the dependence of $\mathcal{E}$ on $\mathcal{Q}$ is given in Eq. (A5).

[40] T. Faulkner and J. Polchinski, Semi-Holographic Fermi Liquids, J. High Energy Phys. 06 (2011) 012.

[41] M. Spradlin and A. Strominger, Vacuum States for AdS(2) Black Holes, J. High Energy Phys. 11 (1999) 021.

[42] M. A. Metlitski and S. Sachdev, Quantum Phase Transitions of Metals in Two Spatial Dimensions. I. Ising-Nematic Order, Phys. Rev. B 82, 075127 (2010). 
[43] D. F. Mross, J. McGreevy, H. Liu, and T. Senthil, Controlled Expansion for Certain Non-Fermi-Liquid Metals, Phys. Rev. B 82, 045121 (2010).

[44] O. DeWolfe, S. S. Gubser, and C. Rosen, Fermi Surfaces in Maximal Gauged Supergravity, Phys. Rev. Lett. 108, 251601 (2012).
[45] S. H. Shenker and D. Stanford, Black Holes and the Butterfly Effect, J. High Energy Phys. 03 (2014) 067.

[46] J. Maldacena, S. H. Shenker, and D. Stanford, A Bound on Chaos, arXiv:1503.01409.

[47] J. M. Luttinger and J. C. Ward, Ground-State Energy of a Many-Fermion System. II, Phys. Rev. 118, 1417 (1960). 